\title{
Extremity Gangrene Caused by HBV-Related Cryoglobulinemia Vasculitis in a Patient with Diabetes - A Case Report
}

\author{
Yunyi Gao (D)' \\ Dawei Chen' \\ Yan $\mathrm{Li}^{\mathrm{I}}$ \\ Huijiao Chen ${ }^{2}$ \\ Xingwu Ran'
}

'Diabetic Foot Care Center, Department of Endocrinology and Metabolism, West China Hospital, Sichuan University, Chengdu, Sichuan, People's Republic of China; ${ }^{2}$ Department of Pathology, West China Hospital, Sichuan University, Chengdu, Sichuan, People's Republic of China
Correspondence: Xingwu Ran

Diabetic Foot Care Center, Department of Endocrinology and Metabolism, West China Hospital, Sichuan University, 37

Guo Xue Lane, Chengdu, Sichuan, 61004I, People's Republic of China Tel +86-28-85422362

Email ranxingwu@।63.com

\begin{abstract}
We presented a case of hepatitis B virus (HBV)-related type III cryoglobulinemia vasculitis (CryoVas) characterized by extremity gangrene in a patient with diabetes. The 60year-old female had a 10 -year history of poorly controlled type 2 diabetes mellitus. She complained of sudden onset pain and swelling of toes which quickly progressed to gangrene, with fingers becoming pain and dark violet. The patient was initially misdiagnosed as diabetic foot (DF). Although DF is one of the common chronic complications of diabetes, it rarely involves the hand. What is more, the ischemic manifestations of the extremity were not consistent with the results of the vascular examination and immune system changes. The patient had Raynaud's phenomenon, arthralgia, and extremity gangrene. Test results showed cryoglobulinemia multiple positive, polyclonal immunoglobulin with rheumatoid factor negative, lower complement 3, leukocytoclastic vasculitis, and HBV infection. HBVrelated type III CryoVas was finally diagnosed, and a conservative therapy strategy was given. Six months after treatment with cyclophosphamide, corticosteroid, nucleoside/nucleotide analog therapy, local debridement, and dressing change, she recovered and kept no recurrence by following up for 30 months. To our knowledge, this is the first report of extremity gangrene caused by HBV-related CryoVas in a diabetic patient.
\end{abstract}

Keywords: HBV, type III cryoglobulinemia vasculitis, extremity gangrene, diabetic foot

\section{Introduction}

Diabetic foot (DF) is one of the most severe and disabling complications of diabetes, in which almost $50 \%$ of ischemic ulcers or gangrene of DF associate with peripheral arterial disease. ${ }^{1}$ Connective tissue diseases, vasculitis, infections, and other diseases are common causes of extremity gangrene. ${ }^{2}$

Cryoglobulinemia vasculitis (CryoVas) characterizes by the deposition of immune complexes in small and medium-sized blood vessels. The hallmark finding related to clinical symptoms is the detection of cryoglobulin in serum. ${ }^{3}$ Cryoglobulin can subdivide into three types based on the immunoglobulin (Ig) content. Type I is monoclonal Ig, commonly IgM or IgG. Mixed cryoglobulinemia comprises both type II and type III. Type II composes monoclonal Ig (usually IgM), polyclonal Ig (mainly IgG), and type III is polyclonal Ig in nature. Mixed cryoglobulinemia is associated with infections (especially hepatitis $\mathrm{C}$ virus), tumors, or autoimmune diseases. ${ }^{3}$ Only $1.2-4 \%$ of hepatitis $\mathrm{B}$ virus (HBV) patients can develop CryoVas. ${ }^{4}$ Purpuric rash is the most common and frequent manifestation of HBV-related CryoVas (approximately $90 \%$ of patients), while gangrene is reported rarely. ${ }^{4,5}$ Herein, we presented a unique patient with HBV-related 
type III CryoVas manifested as extremity gangrene and discussed therapy strategy in detail. To our knowledge, this is the first case reporting extremity gangrene caused by HBV-related CryoVas in a diabetic patient in the literature. This is also the first CryoVas misdiagnosed as DF.

\section{Case Presentation}

A 60-year-old female presented pain and swelling of left second and third toes for one month and blackening for 20 days in 2017. She also had Raynaud's phenomenon, with no limb numb or intermittent claudication. She had type 2 diabetes mellitus (T2DM) poorly managed for ten years and hypertension diagnosed in the local hospital before admission. The pain and gangrene deteriorated, though her blood glucose controlled well. She had no history of smoking, drinking, or infectious disease.

On admission, physical examination revealed gangrenous lesions at the left second and third toes, and the surrounding skin was red without exudation (Figure 1A). The right index finger was purple; the small finger was significant swelling, pain, and dark violet (Figure 1B). Her left anterior tibial artery pulsation weakened, while the dorsal pedis artery could not be palpated. Sensory examination with $10 \mathrm{~g}$ monofilament on feet was regular. Knee jerk and Achilles reflex were normal. Laboratory evaluation showed hemoglobin was $87 \mathrm{~g} / \mathrm{L}$, hepatitis B surface antigen, hepatitis B core antibody, and hepatitis B e antibody were positive, HBV DNA load was $6.06 \mathrm{E}+03 \mathrm{IU} /$ $\mathrm{mL}$. Hepatitis $\mathrm{C}$ virus and human immunodeficiency virus tested were negative (Table 1). Ankle-brachial index (ABI) values were 1.21 on bilateral limbs. Color Doppler ultrasound examination revealed atherosclerosis and segmental occlusions in the left anterior tibial artery. Lower limb angiography demonstrated left anterior tibial artery and peroneal artery occlusions but with well collateral circulation (Figure 1C). Blood flow in the dorsal arteries and plantar arteries was normal. The electrophysiologic study showed peripheral neuropathy.

The patient was initially diagnosed as DF with Wagner grade 4. Her gangrene did not improve after 30 days' treatment, including insulin, low-molecular-weight heparin, alprostadil, and dressing change. Since the patient had Raynaud's phenomenon, atypical ischemic manifestation, and the gangrene kept worse with treatment, further immunological tests were performed and revealed lower serum complement 3 and complement 4 . The anticardiolipin antibody, anti-nuclear antibodies, anti-neutrophil cytoplasmic antibodies, cytomegalovirus, Epstein-Barr virus, and rheumatoid factor were negative. The serum cryoglobulin was positive (Table 1). Serum electrophoresis revealed $4.9 \%$ abnormal $M$ protein. Immunofixation electrophoresis showed polyclonal IgM, IgG bands, and kappa light chain. The results of bone marrow aspiration, biopsy, and SPECT were also negative. After the gangrene stabilized, we removed the necrotic tissues from the wound bed, and the pathology of the necrotic toes was leukocytoclastic vasculitis (Figure 1D). She was finally diagnosed with HBV-related type III CryoVas.

We treated the patient with cyclophosphamide 1.0 g monthly intravenous with a total dose of $5.0 \mathrm{~g}$, and oral prednisone acetate $60 \mathrm{mg} \mathrm{QD},{ }^{3}$ combined with diabetic management and anti-osteoporosis. The prednisone acetate decreased weekly and was then maintained at $5 \mathrm{mg}$ QD before the last dose of cyclophosphamide. Entecavir was used $0.5 \mathrm{mg}$ QD for six months and then changed to tenofovir $300 \mathrm{mg}$ QD for better viral suppression. ${ }^{4,6}$ The local treatment was mainly dressing change after debridement. The finger recovered quickly, and the wound healed within one month. HBV viral load was lower than the reference value after five months, and the serum cryoglobulinemia became persistent negative after six months. Now the patient is treated with tenofovir $300 \mathrm{mg}$ QD, and after 30 months follow-up, there is no symptom and recurrence (Figure 1E and F).

\section{Discussion}

Since diabetic patients are more likely to develop lower extremity atherosclerotic disease-related ulcers and gangrene, clinicians are prone to thinking foot gangrene due to diabetic complications and ignoring other diseases. Lower extremity atherosclerotic disease's clinical manifestations vary across a broad spectrum from asymptomatic to necrosis of the lower extremity, and ABI is often less than 0.90 . Vascular imaging demonstrates arterial stenosis or occlusion without immune system change. ${ }^{7}$ Besides, T2DM is characterized by insulin resistance and relative insulin deficiency, and it is not an autoimmune disease. The mild peripheral artery disease was not consistent with the extremity ischemia but may be a risk factor in this patient. The common causes of cutaneous small-vessel vasculitis include autoimmune connective tissue disease, inflammatory conditions, infection, medication, and malignancy. Considering the atypical manifestation, cryoglobulinemia multiple positive, leukocytoclastic vasculitis, and HBV infection without other signs of infectious, autoimmune, tumors, or hematological disease, finally, we diagnosed the 
A

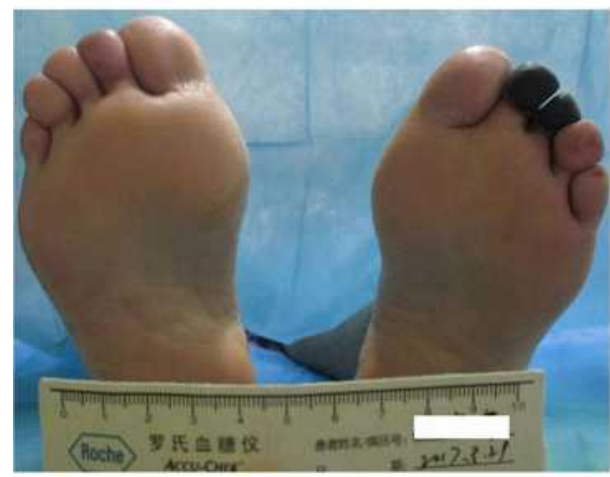

C

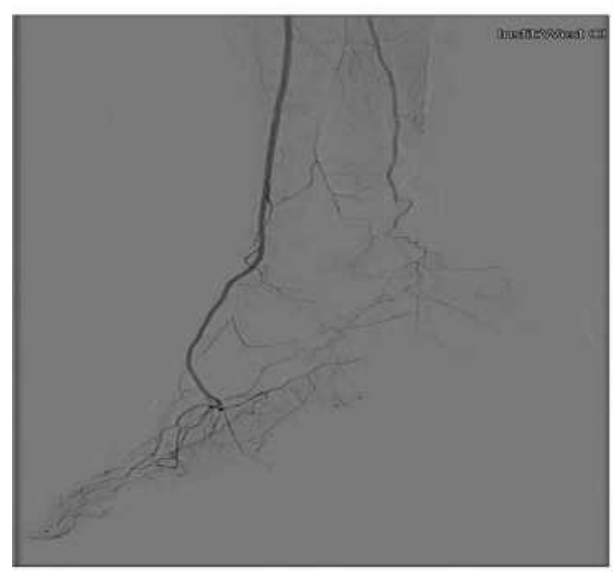

E

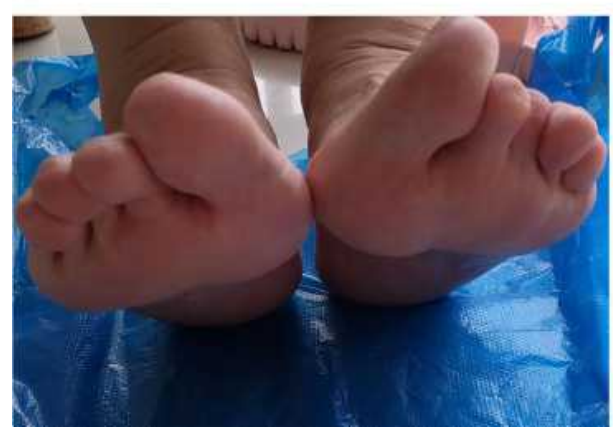

B

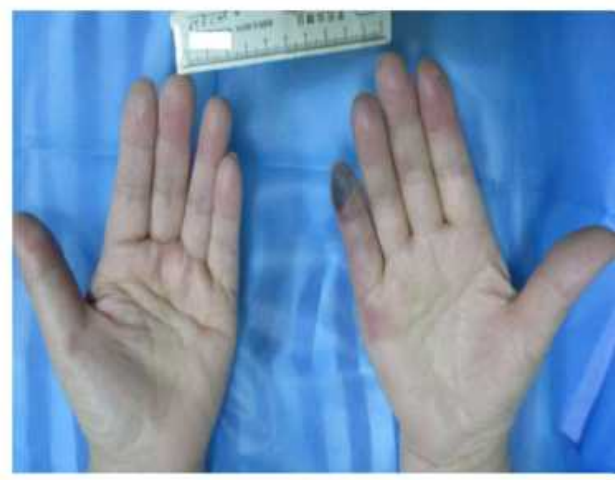

D

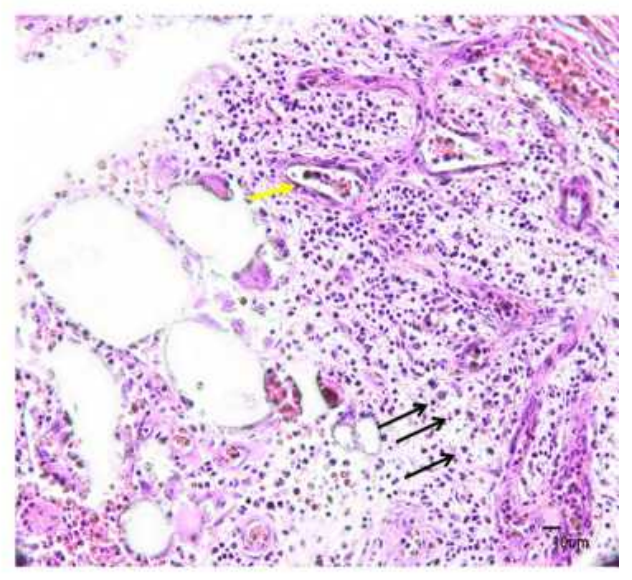

F

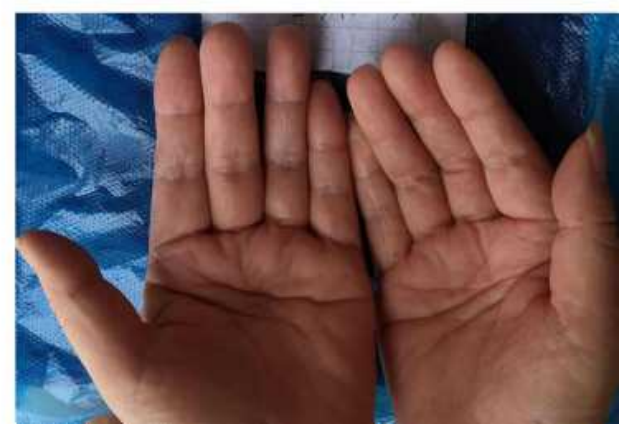

Figure I Variation of the gangrene tissues and histopathologic examination of necrosis extremities. (A and B) Initial presentation of necrotic toes and finger without a defined boundary at admission. (C) Limb angiography of left leg, with well-collateral circulation. (D) Histopathologic examination of necrosis toes with dead blood vessel (yellow arrow), white cells rupture and remained nuclear dust (black arrows), (original magnification $\times 400$ ). (E and F) Toes and fingers healed entirely.

patient as type III CryoVas, and the result of long-term follow-up confirms the diagnosis.

Except for recurrent palpable purpura, joint manifestations, neuropathy, and renal involvement are also frequent symptoms of mixed cryoglobulinemia. ${ }^{3}$ In which peripheral neuropathy and renal dysfunction are common complications of poorly controlled diabetes. Our patient had transient hypertension and apparent renal injury, which were relieved after treatment. This might relate to CryoVas, for renal involvement usually occurs during or shortly after an outbreak of cutaneous vasculitis and manifests with varying degrees of microhematuria, proteinuria, hypertension, and renal failure. ${ }^{8}$ Unfortunately, the renal biopsy was not completed in this case. CryoVas-related neuropathy mainly manifests with paresthesias or burning pain in the legs and muscle weakness. ${ }^{3}$ The patient also 
Table I Laboratory Test Results of the Patient with HBV-Related Type III Cryoglobulinemia Vasculitis

\begin{tabular}{|c|c|c|}
\hline Tests & Results & $\begin{array}{c}\text { Reference } \\
\text { Range }\end{array}$ \\
\hline Hemoglobin, g/dL & 87 & $115-150$ \\
\hline Alanine aminotransferase, IU/L & 9 & $<40$ \\
\hline Aspartate aminotransferase, IU/L & 18 & $<35$ \\
\hline Glycated hemoglobin, \% & 8.0 & $4.5-6.1$ \\
\hline Erythrocyte sedimentation rate, $\mathrm{mm} / \mathrm{h}$ & 68.0 & $<38$ \\
\hline $\mathrm{IL}-6, \mathrm{pg} / \mathrm{mL}$ & 20.49 & $0.00-7.00$ \\
\hline Creatinine, umol/L & 95.0 & $37.0-110.0$ \\
\hline $\begin{array}{l}\text { Estimated glomerular rate } \\
\text { filtration, } \mathrm{mL} / \mathrm{min} / 1.73 \mathrm{~m}^{2}\end{array}$ & 56.26 & $56-122$ \\
\hline Hepatitis B surface antigen & Positive & Negative \\
\hline Hepatitis B e antibody & Positive & Negative \\
\hline Hepatitis B core antibody & Positive & Negative \\
\hline HBV-DNA, IU/mL & $6.06 \mathrm{E}+03$ & $<1.00 \mathrm{E}+02$ \\
\hline Hepatitis $C$ virus & Negative & Negative \\
\hline Human immunodeficiency virus & Negative & Negative \\
\hline Epstein-Barr virus & Negative & Negative \\
\hline Cytomegalovirus & Negative & Negative \\
\hline Urine analysis & Urine protein $(I+)$ & Negative \\
\hline Urine albumin to creatinine ratio, $\mathrm{mg} / \mathrm{g}$ & 54.4 & $<30$ \\
\hline Anticardiolipin antibody & $+1,1: 100$ & Negative \\
\hline Anti-neutrophil cytoplasmic antibodies & Negative & Negative \\
\hline Anticardiolipin antibody & Negative & Negative \\
\hline Rheumatoid factor, IU/mL & $<20$ & $<20$ \\
\hline $\lg A, \mathrm{mg} / \mathrm{L}$ & 2380.00 & $826-2900$ \\
\hline $\lg \mathrm{M}, \mathrm{mg} / \mathrm{L}$ & 3640.00 & $700-2200$ \\
\hline $\lg \mathrm{G}, \mathrm{g} / \mathrm{L}$ & 15.1 & $8.00-15.50$ \\
\hline Complement 3, g/L & 0.8900 & $0.785-1.520$ \\
\hline Complement 4, g/L & 0.2040 & $0.145-0.360$ \\
\hline KAP, g/L & 15.40 & $6.98-13.00$ \\
\hline LAM, g/L & 5.92 & $3.80-6.50$ \\
\hline Cryoglobulins & Positive & Negative \\
\hline Albumin, \% & 51.6 & $55.8-66.1$ \\
\hline Gamma immunoglobulin, \% & 21.4 & $11.1-18.8$ \\
\hline
\end{tabular}

had peripheral neuropathy, but it was difficult to identify with diabetic peripheral neuropathy.

Laboratory results showed increased interleukin 6 (IL-6) in this case. Generally, IL-6 is involved in inflammatory and immunological processes, hematopoiesis, liver, and neuronal regeneration. Elevation of IL-6 signaling is associated with several diseases, including $\mathrm{T} 2 \mathrm{DM},{ }^{9} \mathrm{HBV}^{10}$ and mixed cryoglobulinemia. ${ }^{11}$ The coincidence of T2DM and HBV in this patient may increase the IL- 6 . Although the pathogenesis of IL-6 in the mixed cryoglobulinemia is not confirmed, it plays a role in other vasculitides' pathophysiology, such as Takayasu arteritis and giant cell arteritis. ${ }^{12}$ IL-6 inhibition therapy has already shown remarkable effects on these diseases. Besides, there is a case report for successful treatment of HBV related to cryoglobulinemia vasculitis before. ${ }^{13}$
Approximately $20 \%$ of patients with HBV infection develop extrahepatic manifestations. These are arthritis, glomerulonephritis, uveitis, peripheral neuropathy, Raynaud's phenomenon, Sjögren's syndrome, cutaneous vasculitis, and systemic vasculitis, including polyarteritis nodosa and CryoVas. ${ }^{14}$ CryoVas-related gangrene can be caused by cryoprecipitate, causing microvascular occlusion or vasospasm. Hyperviscosity of the proteins can induce thrombosis and subsequent ischemia in the extremities. ${ }^{15}$ However, our patient did not improve significantly after early anticoagulant and vasodilator therapy.

The individualized therapeutic management approach to HBV-related CryoVas extremity gangrene includes (1) Entecavir was used for antiviral treatment according to existing guidelines. ${ }^{3,6} \mathrm{We}$ changed it to tenofovir for better viral suppression. Monotherapy with nucleoside/nucleotide analog can induce a complete viral suppression and give satisfying clinical outcomes in most mild-to-moderate HBV-related CryoVas patients. ${ }^{4}$ However, for severe HBV-related CryoVas, antiviral treatment may have limited clinical response. ${ }^{16}$ (2) Cyclophosphamide and corticosteroid can reduce the pathogenetic autoimmunelymphoproliferative alterations, autoantibody production and improve tissue lesions. ${ }^{4}$ Our patient was firstly diagnosed with idiopathic CryoVas, and the immunosuppressive treatment failed to suppress $\mathrm{HBV}$ viremia, thus exposing the underlying cause. But there was no standard therapeutic management. ${ }^{4}$ Our treatment is effective, and the long-term low dose corticosteroid was safe with no evidence of iatrogenic hypercortisolism. In this case, it may be stopped earlier for better control of viral replication. (3) Conservative treatment is preferred in local wound care, especially in wounds with unclear etiology. In our view, regular local dressing change is necessary. At the same time, considerate caution should be paid before debridement, vascular surgery, or plastic surgery was taken when the gangrene area is not stable, or CryoVas is not well controlled. If necessary, skin grafting, skin substitutes, and autologous platelet-rich gel can be used to promote the formation of granulation tissue during the repairing period. ${ }^{15}$ Besides, all patients should carefully avoid exposure to the cold and take appropriate protective measures.

\section{Conclusion}

This is the first case of HBV-related type III CryoVas manifested as extremity gangrene in a diabetic patient in the literature. Our long-term exploratory cyclophosphamide, 
corticosteroid, and nucleoside/nucleotide analog treatment successfully control the disease without recurrence in 30 months. But further studies are warranted in this direction. Besides, people with diabetes are more likely to develop ulcers and gangrene than others; if the "diabetes foot" fails to improve after therapy and has atypical manifestations, other diseases relative ulcers need to be considered clinically.

\section{Abbreviations}

ABI, ankle-brachial index; CryoVas, cryoglobulinemia vasculitis; DF, diabetic foot; IL-6, interleukin 6; Ig, immunoglobulin; T2DM, type 2 diabetes mellitus.

\section{Data Sharing Statement}

All datasets generated for this study are included in the Article.

\section{Ethics and Consent}

Written informed consent was obtained from the patient for the publication of any potentially identifiable images or data included in this article.

The report was approved by the ethics committee of the West China Hospital, Sichuan University. (Approved number: No. 542, 2018) prior to study commencement.

\section{Author Contributions}

All authors made substantial contributions to conception and design, acquisition of data, or analysis and interpretation of data; took part in drafting the article or revising it critically for important intellectual content; agreed to submit to the current journal; gave final approval of the version to be published; and agree to be accountable for all aspects of the work.

\section{Funding}

This study was partially supported by West China Nursing Discipline Development Special Fund Project, Sichuan University [Grant No. HXHL20005], the Science and Technology Bureau of Chengdu city [Grant No 2017CY02-00028-GX], Health Medical Big Data Application and Innovation Project in Sichuan [Grant No. 2018gfgw001] and 1.3.5 Project for disciplines of excellence, West China Hospital, Sichuan University [Grant No. ZYGD18025].

\section{Disclosure}

The authors have nothing to disclose.

\section{References}

1. Ibrahim A. IDF clinical practice recommendation on the diabetic foot: a guide for healthcare professionals. Diabetes Res Clin Pract. 2017;127:285-287. doi:10.1016/j.diabres.2017.04.013

2. Roy MK, Datta J, Lahiri D, et al. Digital gangrene an unusual presentation of Takayasu's arteritis. $N$ Am J Med Sci. 2015;7:70-72. doi:10.4103/1947-2714.152082

3. Silva F, Pinto C, Barbosa A, Borges T, Dias C, Almeida J. New insights in cryoglobulinemic vasculitis. $J$ Autoimmun. 2019;105:102313. doi:10.1016/j.jaut.2019.102313

4. Mazzaro C, Dal ML, Visentini M, et al. Hepatitis B virus-related cryogobulinemic vasculitis. The role of antiviral nucleot(s)ide analogues: a review. J Intern Med. 2019;286(3):290-298. doi:10.1111/ joim. 12913

5. Arslan F, Karagöz E, Mert A. Digital ischemic necrosis with cryoglobulinemia associated with hepatitis B infection. J Orthop. 2015;13 (4):448-449. doi:10.1016/j.jor.2015.09.008

6. European Association for the Study of the Liver. EASL 2017 clinical practice guidelines on the management of hepatitis B virus infection. J Hepatol. 2017;67(2):370-398. doi:10.1016/j.jhep.2017.03.021

7. Zhang X, Ran X, Xu Z, et al. Epidemiological characteristics of lower extremity arterial disease in Chinese diabetes patients at high risk: a prospective, multicenter, cross-sectional study. $J$ Diabetes Complications. 2018;32(2):150-156. doi:10.1016/j.jdiacomp.2017.10.003

8. Roccatello D, Fornasieri A, Giachino O, et al. Multicenter study on hepatitis C virus-related cryoglobulinemic glomerulonephritis. Am J Kidney Dis. 2007;49(1):69-82. doi:10.1053/j.ajkd.2006.09.015

9. Akbari M, Hassan-Zadeh V. IL-6 signalling pathways and the development of type 2 diabetes. Inflammopharmacology. 2018;26 (3):685-698. doi:10.1007/s10787-018-0458-0

10. Chen Z, Li YX, Fu HJ, et al. Hepatitis B virus core antigen stimulates IL-6 expression via p38, ERK and NF- $\mathrm{BB}$ pathways in hepatocytes. Cell Physiol Biochem. 2017;41(1):91-100. doi:10.1159/000455954

11. Antonelli A, Fallahi P, Ferrari SM, et al. Parallel increase of circulating CXCL11 and CXCL10 in mixed cryoglobulinemia, while the proinflammatory cytokine IL- 6 is associated with high serum Th2 chemokine CCL2. Clin Rheumatol. 2013;32(8):1147-1154. doi:10.1007/s10067-013-2246-y

12. Yoshifuji H. Pathophysiology of large vessel vasculitis and utility of interleukin-6 inhibition therapy. Mod Rheumatol. 2019;29 (2):287-293. doi:10.1080/14397595.2018.1546358

13. Cohen C, Mekinian A, Saidenberg-Kermanac'h N, et al. Efficacy of tocilizumab in rituximab-refractory cryoglobulinemia vasculitis. Ann Rheum Dis. 2012;71(4):628-629. doi:10.1136/annrheumdis-2011200501

14. Wang CR, Tsai HW. Human hepatitis viruses-associated cutaneous and systemic vasculitis. World J Gastroenterol. 2021;27(1):19-36. doi:10.3748/wjg.v27.i1.19

15. Churchill CL, Polowczyk JR. Cryoglobulinemia: a rare cause of digital gangrene after elective foot surgery. J Foot Ankle Surg. 2017;56:674-679. doi:10.1053/j.jfas.2017.01.041

16. Biasiotta A, Casato M, Cesa SL, et al. Clinical, neurophysiological, and skin biopsy findings in peripheral neuropathy associated with hepatitis C virus-related cryoglobulinemia. J Neurol. 2014;261 (4):725-731. doi:10.1007/s00415-014-7261-7 


\section{Publish your work in this journal}

The Journal of Inflammation Research is an international, peerreviewed open-access journal that welcomes laboratory and clinical findings on the molecular basis, cell biology and pharmacology of inflammation including original research, reviews, symposium reports, hypothesis formation and commentaries on: acute/chronic inflammation; mediators of inflammation; cellular processes; molecular mechanisms; pharmacology and novel anti-inflammatory drugs; clinical conditions involving inflammation. The manuscript management system is completely online and includes a very quick and fair peerreview system. Visit http://www.dovepress.com/testimonials.php to read real quotes from published authors. 